\title{
Direct Extraction of an Admittance Domain Behavioral Model from Large-Signal Load-pull Measurements
}

\author{
M. Rocio Moure ${ }^{1}$, Michael Casbon ${ }^{2}$, Mónica Fernández-Barciela ${ }^{1}$, and Paul J. Tasker ${ }^{2}$ \\ ${ }^{1}$ Universidad de Vigo, Vigo, 36310 ES \\ ${ }^{2}$ Cardiff University, Cardiff, CF24 3AA GB
}

\begin{abstract}
Look-up table behavioral models (i.e. X-parameters, Cardiff model), input drive ' $\left|A_{11}\right|$ ' referenced, extracted directly from measurement data, provide an accurate non-linear CAD modeling solution. Typically, formulated, like s-parameters, in the travelling wave (A-B) domain, since these waves can be directly measured and controlled in the high frequency domain. However, if formulated in the admittance (I-V) domain they would provide a more robust MMIC design modelling solution supporting the capability of width and frequency scaling. Presently, no technique has been presented that allows for the extraction of admittance behavioral models directly from load-pull measurements. Previous solutions have all involved complex indirect procedures based on using an extracted (A-B) domain behavioral model and CAD simulations using voltage sources.

In this paper, a new extraction approach is presented which, by including the influence of variable ' $V_{11}$ ', allows for direct extraction of admittance behavioral models. This approach has been validated on GaN devices.
\end{abstract}

Index Terms - behavioral model, non-linear modeling, GaN FETs, MMIC design, admittance domain.

\section{INTRODUCTION}

Look-up table (LUT) frequency domain behavioral models are commonly used to predict nonlinear behavior of active devices and circuits, providing robust and accurate tools for microwave circuits design. Typically, such behavioral models are formulated in terms of large-signal parameters relating device ports travelling waves (A-B), for example, the PHD model or the Cardiff model [1] - [2].

In the framework of broadband MMIC circuit design, model accuracy and scalability over frequency and transistor width are key requirements. A requirement typically addressed by blackbox state functions (I-V and Q-V) formulated models whether table-based [3] - [4] or compact [5] - [6]. While width scaling is feasible in the (A-B) domain [7], [8], it has been recently demonstrated that if the LUT behavioral model is formulated in the (I-V) admittance domain, it has the capability to also meet both width and frequency scaling objectives [9], [10], [11].

Unfortunately, at high frequency, nonlinear calibrated measurement systems control and measure the (A-B) travelling waves, hence while the extraction of (A-B) formulated behavioral models is straightforward, this is not the case for (I-V) admittance domain based behavioral models. To date, the extraction of these models has not been done directly but via complex indirect/simulation based procedures [9], [10], [11]. For example, initially extracting, directly from measured load-pull data, an extrinsic LUT (A-B) behavioral model, referenced to ' $\left|\mathrm{A}_{1,1}\right|$ '.
CAD simulations may then be performed using this (A-B) model, by applying voltage sources to generate a dataset suitable for the extraction of an (I-V) admittance domain based LUT behavioral model, referenced to ' $\left|V_{1,1}\right|$ '. This procedure makes the extraction a complex and time consuming process, with many steps and possible accumulated errors. But so far, such procedures were required since microwave nonlinear vector network analyzer (NVNA) measurement systems use $50 \mathrm{ohm}$ RF power generators, that only allow the user to directly control the stimulus traveling waves, but would require the application of complex real time iterative procedures to emulate RF voltage generators.

In the new extraction technique proposed in this paper this limitation is overcome. In this technique, during behavioral model extraction from measured waveforms, the ' $\mathrm{V}_{1,1}$ ' dependence -obtained from averaged measurement data- is explicitly considered, thus, allowing for the first time, direct extraction of an LUT admittance (I-V) domain behavioral model from (A-B) measurements.

\section{EXtraction of A-B Behavioral Model}

The role of the LUT behavioral model is to provide a formulation that can both describe the nonlinear device measured behavior and provide an accurate tool for CAD of non-linear MMICs. To achieve the first goal, the model must be able to accurately replicate the measured large-signal device performance as a function of drive level and source/load impedance variations. The second goal requires a model formulation that is robust with respect to interpolation capabilities and with some added "intelligence" for reasonable extrapolation.

These goals are achieved, for example, in the Cardiff model by using a formulation to describe the response of a non-linear system based on the mixing interactions of all the input stimuli. In this paper, we will use this model to describe the proposed extraction technique.

\section{A. The traditional Cardiff model formulation}

Typically, since measurements are performed by stepping the input drive level, hence during LUT model extraction the value of ' $\left|A_{1,1}\right|$ ' is assumed constant, the generated model is stored as an indexed LUT of model parameters ' $\mathrm{M}_{\mathrm{phi}}$ ' vs ' $\left|\mathrm{A}_{1,1}\right|$ '. In the case of using measured load-pull data to predict fundamental and second harmonic device behavior, it is the input stimuli ' $A_{2,1}$ ' and ' $A_{2,2}$ ', that need to be accounted for in the model 

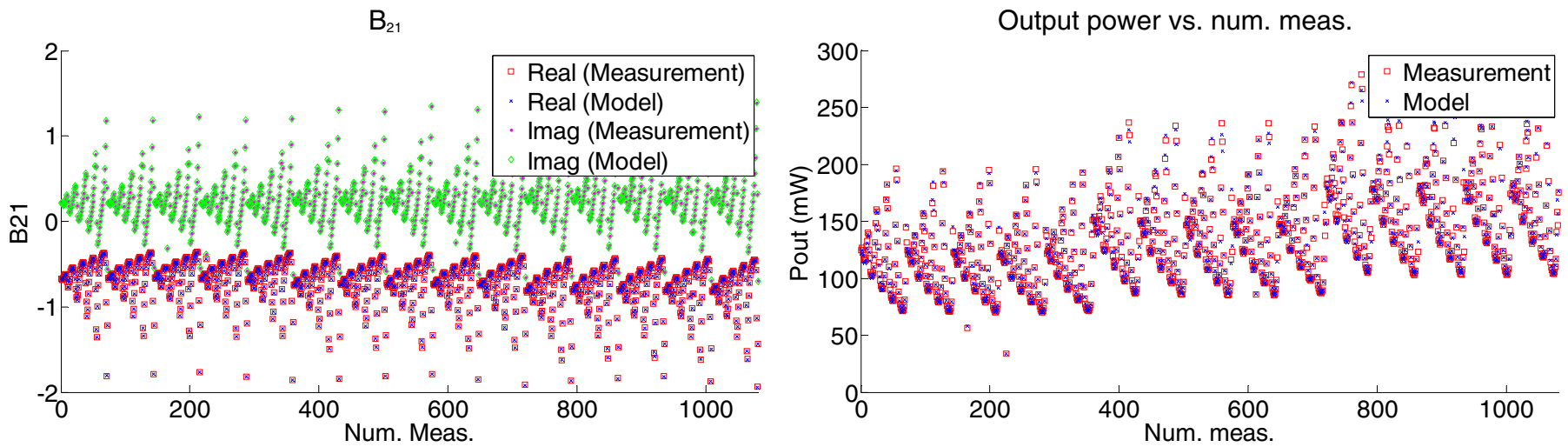

Fig. 1. Results for a 100 um gate width $\mathrm{GaN}$ device with non-constant $\mathrm{A}_{11}$ at the input ( $\mathrm{P}_{\text {in }}$ set to 8,9 and $10 \mathrm{dBm}$ ). $\mathrm{P}_{\text {in }}$ average value, $9.2 \mathrm{dBm}$. Bias point: $\mathrm{Vgs} 0=-2.45 \mathrm{~V}, \mathrm{Vds} 0=25 \mathrm{~V}$. Fundamental freq. $4 \mathrm{GHz}$. a) Real and imaginary parts of $\mathrm{B}_{21}$. b) Fundamental output power, in mW. Comparison between all the measurement points and the extracted model predictions. Output is referenced to the intrinsic plane.

formulation. Eq. (1) shows the required Cardiff model formulation, in this case, after phase normalization.

$$
B_{p, h}=\sum_{i=0}^{W} M_{p, h . i}\left(\left|A_{1,1}\right|\right)\left|A_{2,1}\right|^{a_{i}} \angle A_{2,1}^{b_{i}}\left|A_{2,2}\right|^{c_{i}} \angle A_{2,2}^{d_{i}}
$$

Where ' $p$ ' refers to the device port number and ' $h$ ' refers to the harmonic number. The model complexity is determined by ' $\mathrm{W}$ ', the number of model coefficients ' $\mathrm{M}_{\mathrm{p}, \mathrm{h}, \mathrm{i}}$ ' The index ' $\mathrm{i}$ ' is associated with the selected mixing coefficients $\left(a_{i}, b_{i}, c_{i}\right.$ and $\left.\mathrm{d}_{\mathrm{i}}\right)$. Note, the mixing theory requires $\mathrm{a}_{\mathrm{i}}=\left|\mathrm{b}_{\mathrm{i}}\right|+2 \mathrm{r}_{\mathrm{i}}$ and $\mathrm{c}_{\mathrm{i}}=\left|\mathrm{d}_{\mathrm{i}}\right|+2 \mathrm{~s}_{\mathrm{i}}$, where ' $r_{i}$ ' and ' $\mathrm{s}_{\mathrm{i}}$ ' are stepped integer quantities, $0,1,2 \ldots$ This modelling approach, thus transforms the measured, fundamental and second harmonic large-signal load-pull data into an ' $\left|\mathrm{A}_{1,1}\right|$ ' indexed LUT of model parameters ' $\mathrm{M}_{\mathrm{p}, \mathrm{h}, \mathrm{i}}$ '. But this approach, defined in this way, cannot be applied to NVNA measurements when the input drive level is not constant. This would be the case for a non $50 \Omega$ input impedance measurement system, during intrinsic model extraction, due to the de-embedding

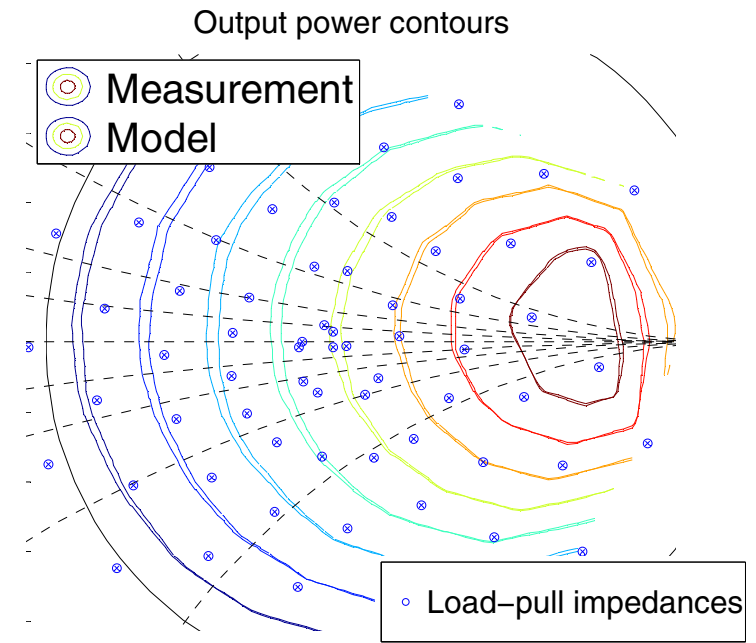

Fig. 2. Results for a 100 um gate width $\mathrm{GaN}$ device with non-constant $\mathrm{A}_{11}$ at the input ( $P_{\text {in }}$ set to 8,9 and $\left.10 \mathrm{dBm}\right) . P_{\text {in }}$ average value, $9.2 \mathrm{dBm}$. Bias point: $\mathrm{Vgs} 0=-2.45 \mathrm{~V}, \mathrm{Vds} 0=25 \mathrm{~V}$. Fundamental freq. $4 \mathrm{GHz}$. Output power contours around the optimum load. Comparison between all the measurement points and the extracted model predictions. Output is referenced to the intrinsic plane. process, or if measured parameters are directly transformed from the $(A-B)$ to the $(I-V)$ domain.

\section{B. Advanced formulation for Cardiff model extraction}

To solve the previous limitation, we propose an advanced formulation, in which the input stimuli ' $A_{1,1}$ ' is also included in the mixing formulation, for use during Cardiff model extraction. Eq. (2) shows the modified (advanced) Cardiff model formulation, after phase normalization, now required, that includes all input stimuli ' $\mathrm{A}_{1,1}$ ', ' $\mathrm{A}_{2,1}$ ' and ' $\mathrm{A}_{2,2}$ '.

$B_{p, h}=$
$\sum_{t=0}^{V} \sum_{i=0}^{W} K_{p, h ., i, t}\left|A_{2,1}\right|^{a_{i}} \angle A_{2,1}^{b_{i}}\left|A_{2,2}\right|^{c_{i}} \angle A_{2,2}^{d_{i}}\left|A_{1,1}\right|^{e_{i, t}}$

Using this formulation, extraction with a variable ' $\left|\mathrm{A}_{1,1}\right|$ ' is now possible. First, the ' $\mathrm{K}_{\mathrm{p}, \mathrm{h}, \mathrm{i}, \mathrm{t}}$ ' model coefficients, with corresponding mixing order given by ' $\mathrm{a}_{\mathrm{i}}+\mathrm{b}_{\mathrm{i}}+\mathrm{e}_{\mathrm{i}, \mathrm{t}}$ ', are extracted directly from the measured data. The required LUT ' $\mathrm{M}_{\mathrm{p}, \mathrm{h}, \mathrm{i}}$ ' model coefficients, are then computed for the mean value of ' $\left|\mathrm{A}_{1,1}\right|$ ', as follows:

$$
M_{p, h . i}\left(\left|A_{1,1}\right|\right)=\sum_{t=0}^{V} K_{p, h . i, t}\left|A_{1,1}\right|^{e_{i, t}}
$$

Also, using this formulation, determination of the required model complexity (constrained by the maximum mixing order and maximum value of mixing coefficients $r_{i}, b_{i}, s_{i}, d_{i}$ and $e_{i, t}$ ) can now, for the first time, be automated. The new mixing coefficient ' $\mathrm{e}_{\mathrm{i}, \mathrm{t}}$ ' of ' $\left|\mathrm{A}_{1,1}\right|$ ' is defined as ' $\mathrm{e}_{\mathrm{i}, \mathrm{t}}=\left|\mathrm{f}_{\mathrm{i}}\right|+2 \mathrm{t}$ ', where ' $\mathrm{f}_{\mathrm{i}}$ ' is computed from the mixing relationship to the harmonic ' $h$ '. In this case ' $\mathrm{f}_{\mathrm{i}}=\mathrm{h}-\left(\mathrm{b}_{\mathrm{i}}+2 \mathrm{c}_{\mathrm{i}}\right)$ '. While ' $\mathrm{t}$ ' is another stepped integer quantity, $0,1,2 \ldots$ Higher values imply a higher mixing order.

This new method allows for the extraction of the (A-B) domain behavioral model (as defined in (1)) from data sets when there is non-constant input level. This new formulation is, at least, valid for small perturbations of ' $\left.\left|\mathrm{A}_{1,1}\right|\right|^{\prime}$, hence low mixing order values; i.e. ' $\mathrm{t}<2$ '.

For testing the feasibility of the proposed model extraction procedure, an (A-B) domain behavioral model was now extracted from NVNA measured load-pull datasets, both fundamental and second harmonic, for a non-constant input drive 

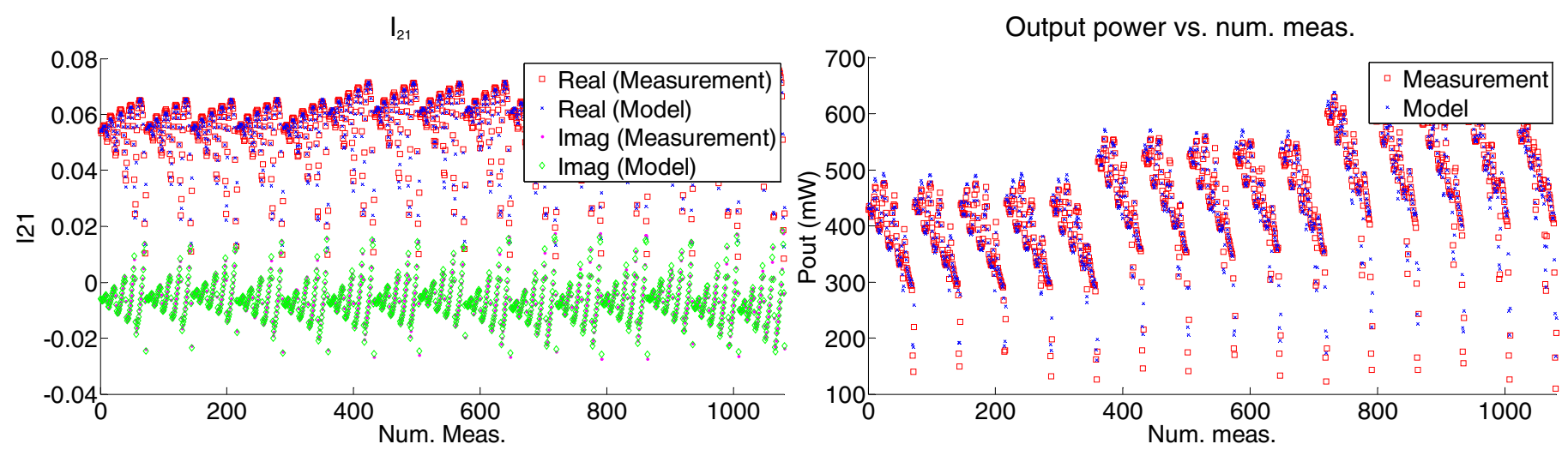

Fig. 3. Results for a $200 u m$ GaN device with non-constant $A_{11}$ at the input ( $P_{\text {in }}$ set to 10,11 and $12 \mathrm{dBm}$ ). $P_{\text {in }}$ average value, 10.9dBm. Bias point: $V$ gs $0=-$ $2.45 \mathrm{~V}, \mathrm{Vds} 0=25 \mathrm{~V}$. Fundamental freq. 4GHz. a) Real and imaginary parts of B21. b) Fundamental output power, in mW. Comparison between all the measurement points and the extracted model predictions. Output is referenced to the intrinsic plane.

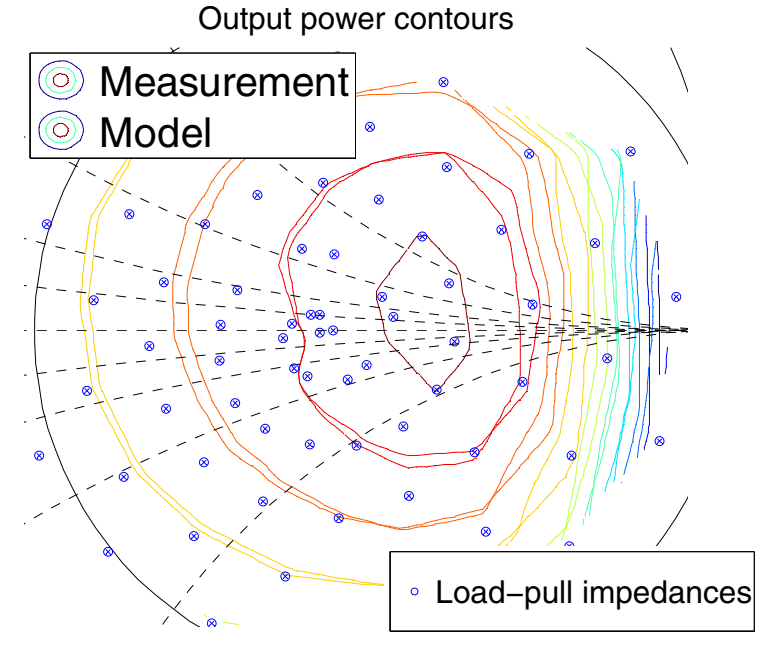

Fig. 4. Results for a $200 \mathrm{um}$ GaN device with non-constant $\mathrm{A}_{11}$ at the input ( $P_{\text {in }}$ set to 10,11 and $12 \mathrm{dBm}$ ). $P_{\text {in }}$ average value, $10.9 \mathrm{dBm}$. Bias point: $\mathrm{Vgs} 0=-$ $2.45 \mathrm{~V}, \mathrm{Vds} 0=25 \mathrm{~V}$. Fundamental freq. $4 \mathrm{GHz}$. Output power contours around the optimum load. Comparison between all the measurement points and the extracted model predictions. Output is referenced to the intrinsic plane

level. In this case, three input power levels from a dataset generated, on a $1 \mathrm{~dB}$ grid, was used. Figs. 1 and 2 summarize the robustness of the achieved results on a 100um gate width GaN device at $4 \mathrm{GHz}$ fundamental frequency. The NVNA active fundamental load-pulling is performed around the optimum load impedance, and the second harmonic load-pulling is performed around the Smith Chart edge. In this case, the required mixing coefficients $\left(\mathrm{r}_{\mathrm{i}}, \mathrm{b}_{\mathrm{i}}, \mathrm{s}_{\mathrm{i}}, \mathrm{d}_{\mathrm{i}}\right.$ and $\left.\mathrm{t}\right)$ are determined using the following constraints, a maximum mixing order of 7 and maximum values for mixing coefficients being $\mathrm{b}=2, \mathrm{r}=1, \mathrm{~d}=1, \mathrm{~s}=0$ and for ' $\left|\mathrm{A}_{1,1}\right|$ ' $\mathrm{t}=1$. Using this approach, an LUT $(A-B)$ behavioral model extraction has been successfully undertaken using NVNA measured datasets obtained from non-constant input drive levels.

\section{Model EXTENSION to the AdmitTANCE Domain AND DIRECT EXTRACTION VALIDATION}

Consider now the translation of the proposed advanced extraction formulation (2) to the (I-V) admittance domain, as shown in (4):

$$
I_{p, h}=\sum_{t=0}^{V} \sum_{i=0}^{W} L_{p, h . i, t}\left|V_{2,1}\right|^{a_{i}} \angle V_{2,1}^{b_{i}}\left|V_{2,2}\right|^{c_{i}} \angle V_{2,2}^{d_{i}}\left|V_{1,1}\right|^{e_{i, t}}
$$

This new formulation allows for the (I-V) admittance domain model extraction directly from waveform measurement data, even when the value of ' $\left|\mathrm{V}_{1,1}\right|$ ' is not constant, hence, addressing the limitations imposed by NVNA-based measurement systems based on microwave power sources. The required (I-V) admittance behavioral LUT ' $\mathrm{N}_{\mathrm{p}, \mathrm{h}, \mathrm{i}}$ ' model coefficients (from the translation to the admittance domain of (1)) can be also computed for the mean value of ' $\left|\mathrm{V}_{1,1}\right|$ ', as follows:

$$
N_{p, h . i}\left(\left|V_{1,1}\right|\right)=\sum_{t=0}^{V} L_{p, h . i, t}\left|V_{1,1}\right|^{e_{i, t}}
$$

Figs. 3 and 4 show the success of the proposed procedure, again using three input power levels, from a dataset generated from on a $1 \mathrm{~dB}$ grid, to accurately extract (I-V) behavioral model. For this example, a 200um gate width $\mathrm{GaN}$ device is selected. The maximum mixing order and the maximum values for mixing coefficients are the same as defined in II. The robustness of the extracted (I-V) admittance model also when compared to the traditional (A-B) domain model, is further highlighted in figs. 5 and 6 , via examples at higher frequencies and/or increased device width. Note that the reference parameters in each model, the mean values of ' $\mathrm{V}_{1,1}$ ' for the (I-V) admittance domain model, and ' $\mathrm{A}_{1,1}$ ' for the (A-B) travelling wave domain model, are not fully equivalent, due to the variation of ' $\mathrm{V}_{1,1}$ ' during the measurements.

In this section, we have shown that even after the "measured" behavioral model parameters are transformed in "real time" from the (A-B) domain to the (I-V) domain, an accurate nonlinear admittance behavioral model can be generated, thus 


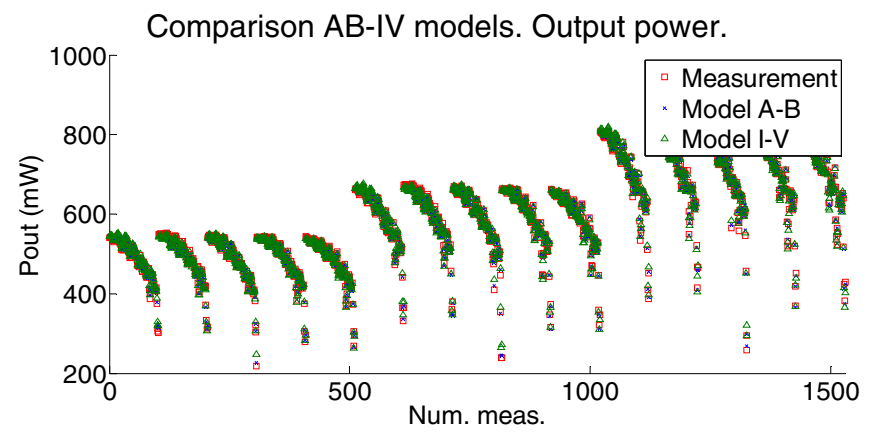

Fig. 5. Results for a 300um GaN device with non-constant A11 at the input (Pin set to $18.5,19.5$ and $20.5 \mathrm{dBm}$ ). Pin average value, $19.5 \mathrm{dBm}$. Bias point: $\mathrm{Vgs} 0=-2.45 \mathrm{~V}, \mathrm{Vds} 0=25 \mathrm{~V}$. Fundamental output power, in $\mathrm{mW}$ at $16 \mathrm{GHz}$. Again, A-B and I-V models are in very close agreement. Output is referenced to the intrinsic plane

demonstrating a direct technique to obtain admittance domain behavioral models from NVNA measured data.

This new method reduces significantly the extraction time for the (I-V) admittance domain model, in comparison to the original method used and described in the introduction section. This is mainly because the model coefficients extraction is now directly done from measurements in one processing step, without involving a simulator. It is eliminating the need of additional intermediate simulations step, as in the previous approaches [9], [10] which provides for a significant time saving. Basically, the time it now takes to extract and (I-V) admittance domain model is identical to that for extraction of the original (A-B) travelling wave domain model.

\section{CONCLUSIONS}

This manuscript provides a robust and accurate solution for the extraction of LUT (I-V) admittance domain behavioral models directly (analytically), for the first time, from measured large-signal waveform load-pull data. Admittance domain models are very useful, since they provide for a much simpler implementation within the CAD tools, which is more compatible with supporting scalability over frequency and transistor size, hence providing a behavioral modelling framework more flexible and suitable for broadband nonlinear MMIC design.

\section{ACKNOWLEDGMENT}

This work has received financial support from the Spanish National Board of Scientific and Technology Research and the European Union (European Regional Development FundERDF), through Project TEC2014-60283-C3-3-R, Xunta de Galicia \& ERDF through "Agrupación Estratéxica Consolidada de Galicia accreditation 2016-2019” and Xunta de Galicia (Consellería de Cultura, Educación e Ordenación Universitaria) $\&$ ESF (European Social Fund) through galician universities predoctoral grant.

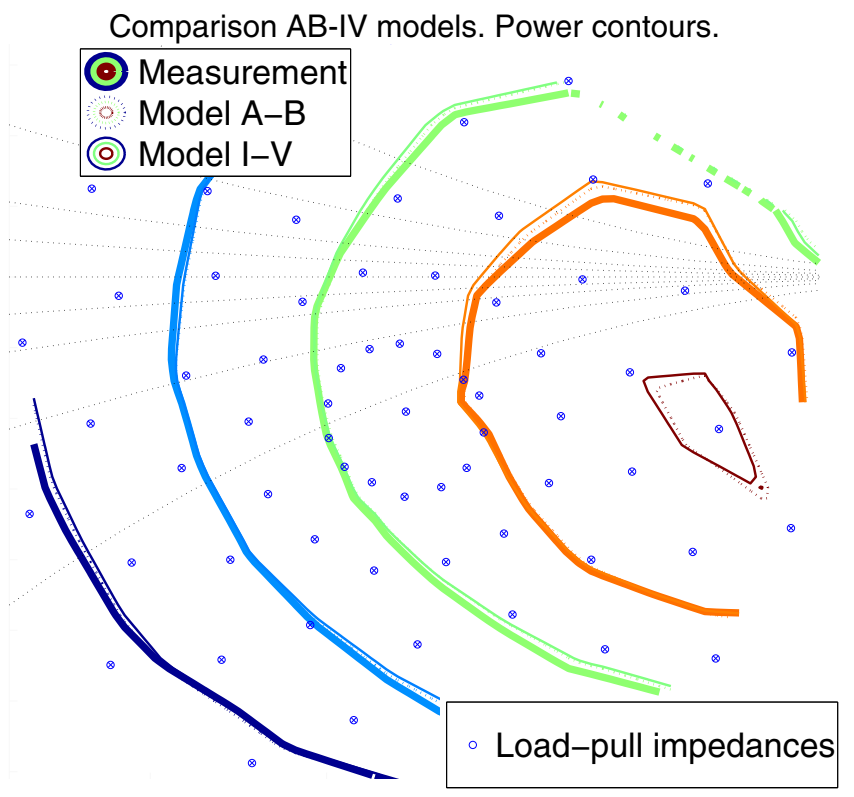

Fig. 6. Results for a $100 \mathrm{um} \mathrm{GaN} \mathrm{device} \mathrm{with} \mathrm{non-constant} \mathrm{A}_{11}$ at the input ( $P_{\text {in }}$ set to $8.14,9.14$ and $\left.10.14 \mathrm{dBm}\right) . P_{\text {in }}$ average value, $9.14 \mathrm{dBm}$. Bias point: $\mathrm{Vgs} 0=-2.45 \mathrm{~V}, \mathrm{Vds} 0=25 \mathrm{~V}$. Fundamental output power contours at $8 \mathrm{GHz}$. A$\mathrm{B}$ and I-V models are in very close agreement. Output is referenced to the

\section{REFERENCES}

[1] J. Verspecht, D.E. Root, "Polyharmonic distortion modeling", IEEE Microwave Mag., vol. 7, no. 3, pp. 44-57, June 2006.

[2] S. Woodington et al., "A novel measurement based method enabling rapid extraction of a RF waveform look-up table based behavioral model", IEEE MTT-S Int. Microwave Symp. Dig., pp. 1453-1456, June 2008.

[3] D. E. Root, S. Fan, and J. Meyer, "Technology independent large-signal FET's models: A measurement-based approach to active device modeling,"in Proc. 15th ARMMS Conf., 1991, pp. 1-21.

[4] M. Fernández-Barciela et al., "A simplified broad-band large-signal nonquasi-static table-based FET model", IEEE Trans. Microw. Theory Tech., vol. 48, no. 3, pp. 395-405, Mar. 2000.

[5] J. Xu et al., "Dynamic FET Model-DynaFET-for GaN Transistors from NVNA Active Source Injection Measurements", Int. Microw. Symp. Dig. June, 2014

[6] I. Angelov et al., "Extension of the Chalmers non linear HEMT and MESFET model", IEEE Trans. Microw. Theory and Tech., vol. 44, no. 10-1, pp. 1664-1674, Oct. 1996.

[7] D.E. Root et al., "Scaling of X-parameters for device modeling", IEEE MTT-S Int. Microw. Symp. Dig., pp. June 2012.

[8] J.G. Leckey, "A scalable X-parameter model for GaAs and GaN FETs", European Microw. Integrated Circuits Conf., 2011

[9] M. Koh et al., "Frequency Scalable Large Signal Transistor Behavioral Model based on Admittance Domain Formulation", IEEE MTT-S Int. Microw. Symp. Dig., pp. June 2014.

[10] M. R. Moure et al.," Broadband Non-Linear FET Behavioral Model Defined in the Admittance Domain", EuMW. European Microw. Integrated Circuits Conf., Oct 2016.

[11] D. E. Root et al., "Frequency-scalable nonlinear behavioral transistor model from single frequency X-parameters based on time-reversal transformation properties", Microw. Measurement Conf., ARFTG. Dec. 2015. 